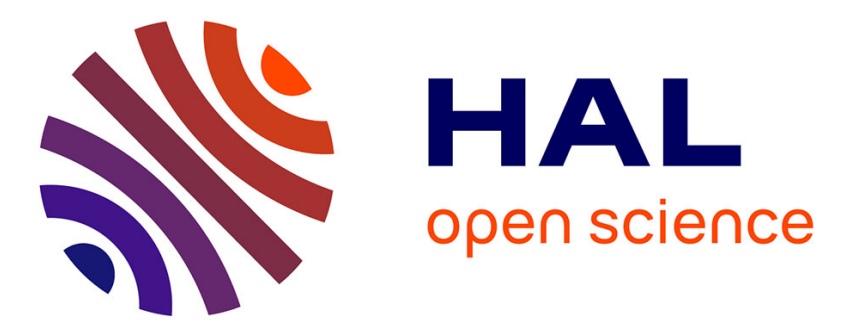

\title{
Capturing the Cumulative Effect in the Pump Probe Transient Thermoreflectance Technique using Network Identification by Deconvolution Method
}

\author{
Y Ezzahri, G. Pernot, Karl Joulain, A. Shakouri
}

\section{To cite this version:}

Y Ezzahri, G. Pernot, Karl Joulain, A. Shakouri. Capturing the Cumulative Effect in the Pump Probe Transient Thermoreflectance Technique using Network Identification by Deconvolution Method. Material Research Society Spring Meeting 2011, Apr 2011, San Francisco, CA, United States. pp.mrss111347-bb07-05, 10.1557/opl.2011.1495 . hal-01537859

\section{HAL Id: hal-01537859 \\ https://hal.univ-lorraine.fr/hal-01537859}

Submitted on 14 Jun 2017

HAL is a multi-disciplinary open access archive for the deposit and dissemination of scientific research documents, whether they are published or not. The documents may come from teaching and research institutions in France or abroad, or from public or private research centers.
L'archive ouverte pluridisciplinaire HAL, est destinée au dépôt et à la diffusion de documents scientifiques de niveau recherche, publiés ou non, émanant des établissements d'enseignement et de recherche français ou étrangers, des laboratoires publics ou privés.

\section{(1)(1) $\$(0)$}

Distributed under a Creative Commons Attribution - NonCommercial - ShareAlikel 4.0 


\title{
Capturing the Cumulative Effect in the Pump Probe Transient Thermoreflectance Technique using Network Identification by Deconvolution Method
}

\author{
Y. Ezzahri, ${ }^{1 *}$ G. Pernot ${ }^{2}$, K. Joulain ${ }^{1}$ and A. Shakouri ${ }^{2}$
}

${ }^{1}$ Institut Pprime, CNRS-Université de Poitiers-ENSMA, Département Fluides, Thermique, Combustion, ENSIP-Bâtiment de mécanique, 2 rue Pierre Brousse, F 86022 Poitiers, Cedex, France.

${ }^{2}$ Department of Electrical Engineering, University of California at Santa Cruz, 1156 High street, Santa Cruz, California, 95064, USA.

*younes.ezzahri@univ-poitiers.fr

\begin{abstract}
Network Identification by Deconvolution (NID) method is used to capture the heat cumulative effect in the homodyne configuration of the Pump-Probe Transient Thermoreflectance (PPTTR) experiment. This cumulative effect is very important in the interpretation of the PPTTR which is becoming widely used for the extraction of thin film thermal conductivity. We show that this intrinsic behavior can be introduced as a cumulative effect weight function in the time constant spectrum of the structure under study. We show how the main features of this weight function change when we change the laser repetition rate and/or the laser pump beam modulation frequency, and how these changes may affect the extraction of the thermal properties of the sample under study, particularly the thermal conductivity and the interface thermal resistance. Numerical simulations of the PPTTR experiment are used to validate the application of NID method. Limitations of the method will also be discussed.
\end{abstract}

\section{INTRODUCTION}

Pump-Probe Transient Thermoreflectance (PPTTR) is an interesting technique whose first utilization to study thermal transport experimentally was reported by Paddock and Eesley [1]. For nearly two decades, PPTTR technique has been an effective tool for studying thermal transport in thin films, low dimensional structures (multilayers and superlattices) [2] and recently liquids [3]. In contrast to the $3 \omega$ method [4], PPTTR allows for the distinction between the thermal conductivity of thin film and the metal transducer-semiconductor interface thermal resistance [2]. In this technique, an intense short laser pulse "pump" is used to heat the film, and a delayed weak (soft) short laser pulse "probe" is used to monitor the top free surface reflectivity change induced by the cooling of the thin film after absorption of the pump pulse. The pump and probe can come from the same primary laser oscillator source, a configuration called homodyne PPTTR [5], or they can be issued from two locked laser oscillator sources, a configuration called heterodyne PPTTR [6]. Because in a PPTTR technique, high laser repetition rates are generally used, in many situations, the system under study does not have sufficient time to return to its equilibrium state between two successive laser pulses. The effect of the multiple pulses accumulates and the measured thermal decay can significantly differ from the response to as single pulse. Many authors have tackled the question of the accumulation phenomenon in PPTTR experiments in the past. The cumulative effect was first described by Capinski and Maris [7] in terms of the impulse response of the sample in the time domain assuming a onedimensional thermal transport model. Later Cahill [8] gave an equivalent description in the frequency domain by taking into account radial heat transfer in layered isotropic media. Cahill's intuitive idea to analyze the ratio of in-phase and out-of-phase signals, which is referred to as 
time-domain transient thermoreflectance (TDTR), is now becoming widely used to extract thin film thermal conductivity. Recently Schmidt et al [9] have extended the analysis to the case of anisotropic layered media.

In a recent work, we showed that Network Identification by Deconvolution (NID) method has a great potential to be adapted and used to analyze the thermal signal in a PPTTR experiment [10]. The main advantage is that NID is a direct solution for the inverse heat conduction problem and it does not require a specific layer structure to fit the experimental results. However the previous analysis was restricted to the case of the inherent impulse response of the sample and neglected the accumulation effect. This occurs when one uses low repetition rate pulsed lasers in which case the time between two successive laser pulses, $T$, becomes large enough compared to the main thermal time constant of the sample. The purpose of this paper is to extend the NID analysis to shed light on the accumulation phenomenon of the PPTTR set up in the homodyne configuration. We show how this might affect the extraction of thermal properties and the interpretation of the results.

\section{CUMULATIVE WEIGHT FUNCTION}

In a PPTTR experiment, the duration of the optical pulses produced by the laser source (e.g. Ti:Sapphire femtosecond laser) are much shorter than the time scales of interest in the thermal model so that we can approximate the train of ultrashort laser pulses by a Dirac delta comb function. In the homodyne configuration of the PPTTR technique, the main laser output is split into two trains; the first intense pulsed laser beam "the pump" is modulated at a reference frequency $\omega_{m}$, while the second soft pulsed laser beam "the probe" is separated in time with respect to the pump by an adjustable delay $t$. At each time delay, the probe pulses are reflected off the sample under study into a photodetector with an intensity proportional to the surface temperature of the sample induced by the pump pulses. A lock-in detection is used to extract only the components of the measured signal in an extremely narrow band around the reference signal modulation. An interesting review of the essential elements and principle details of the homodyne configuration of the PPTTR can be found in Schmidt et al [9] and Stevens et al [11].

The lock-in amplifier measures the fundamental component of the probe signal at the modulation frequency $\omega_{m}$ and rejects all other harmonics. The output could be either the amplitude and phase or the real and imaginary parts of the signal. Mathematically the solution can be represented in two ways. The first given by Capinski and Maris [7] is in terms of the impulse response of the sample in the time domain, and the second equivalent one given by Cahill [8] is in terms of the response in the frequency domain. Here we consider the time domain expression of the PPTTR signal seen by the lock-in amplifier in the homodyne configuration that can be written as:

$$
S\left(\omega_{m}, t\right)=\frac{\Gamma Q_{P} Q_{S}}{T} \sum_{q=0}^{\infty} h(t+q T) e^{-i \omega_{m}(t+q T)}
$$

Where $Q_{P}, Q_{S}$ and $t$ are the energy per pump pulse, energy per probe pulse, and time delay between the pump and the probe, respectively. $h$ represents the impulse response of the system that is assumed to be linear and time invariant (LTI) and $\Gamma$ is a phenomenological constant that includes the thermoreflectance coefficient, the gain of the different electronics (photodiode, 
resonant filter, lock-in amplifier, etc.) as well as the time constant of the lock-in amplifier. $T=F^{-1}$ is the period of the laser oscillator source where $F$ is the repetition rate, and $\omega_{m}=2 \pi f_{m}$ where $f_{m}$ is the modulation frequency. We multiply Eq. (1) by the factor $e^{i \omega_{m} t}$, then we integrate the real part of the product and apply the mathematical procedure of NID approach $[10,12]$ to this integral function. We get the following result in the logarithmic time $z=\log (t)$ domain:

$$
\left\{\begin{array}{l}
\frac{d Y\left(\omega_{m}, z\right)}{d z}=\Lambda R^{*}(z) \otimes W(z) \text { where } W(z)=\exp [z-\exp (z)] \\
R^{*}(\xi)=R(\xi) \times \Phi(\xi) \text { with } \Phi(\xi)=\frac{1-\cos \left(\omega_{m} T\right) \exp \left(-T e^{-\xi}\right)}{1-2 \cos \left(\omega_{m} T\right) \exp \left(-T e^{-\xi}\right)+\exp \left(-2 T e^{-\xi}\right)} \text { where } \xi=\log \tau
\end{array}\right.
$$

In this expression $\otimes$ represents the symbol of convolution. After deconvolution with the fixed weight function $W(z)$, the result will be a time constant spectrum $R^{*}$ that is the natural product of the impulse time constant spectrum $R$ and a weight function $\Phi$ that describes the cumulative effect of the homodyne PPTTR technique. This Cumulative Weight Function (CWF) is independent of the properties of the sample under study and is function only of the intrinsic properties of the PPTTR set up experiment; the period of the pulsed laser oscillator source and the modulation frequency of the laser pump beam.

\section{DISCUSSION}

The behavior of the CWF as a function of the logarithmic time is reported in Figs 1, for different laser repetition rates $F$ [Fig 1(a)] and modulation frequencies $f_{m}$ (Fig 1(b)], respectively. CWF shows a very interesting bell shape feature. The CWF tends to 1 as $\xi \rightarrow-\infty(\tau \rightarrow 0)$ which corresponds to very short time scales, while it tends to $1 / 2$ as $\xi \rightarrow+\infty(\tau \rightarrow+\infty)$, which corresponds to very long time scales.
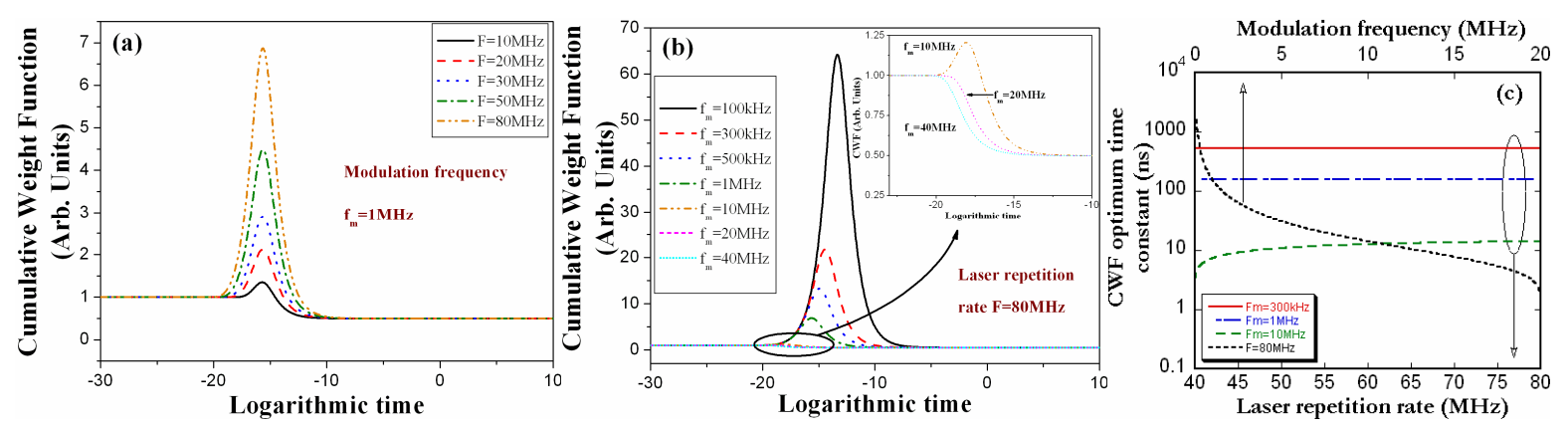

Figure 1 : Variation of the cumulative weight function (CWF) as a function of the logarithmic time for different values of the pulsed laser source repetition rate $F$ (a) and the laser pump beam modulation frequency $f_{m}(\mathrm{~b})$. The inset in (b) represents the behavior of the CWF for large values of $f_{m}$. (c) shows the variation of the optimum time constant $\tau_{o p}$ of the CWF as a function of $F$ for different values of $f_{m}$ and as a function of $f_{m}$ for $F=80 \mathrm{MHz}$. 
An important feature of the CWF is the existence of an optimum time constant $\tau_{o p}$ that corresponds to the maximum amplitude. $\tau_{o p}$ can be obtained easily by calculating the derivative of $\Phi$ and making it equal zero. The analytical expression of $\tau_{o p}$ is given by:

$$
\xi_{o p}=\log \left[T\left[\operatorname{arcosh}\left(\cos \left(\omega_{m} T\right)^{-1}\right)\right]^{-1}\right] \Rightarrow \tau_{o p}=T\left[\operatorname{arcosh}\left(\cos \left(\omega_{m} T\right)^{-1}\right)\right]^{-1}
$$

Where "arcosh" refers to the inverse hyperbolic cosine function.

As we can see in Fig 1(a), for a fixed $f_{m}$, decreasing $F$ results in a considerable change in both the maximum amplitude and width of the CWF. While the position of the optimum time constant $\tau_{o p}$ stays constant, the maximum amplitude decreases and the bell shape of the CWF curve shrinks as we decrease $F$. On the other hand, when $F$ is fixed, we see in Figs $1(b)$ that, by increasing $f_{m}$, the maximum amplitude and the width of the bell shape curve of the CWF decrease. Furthermore, the position of the optimum time constant $\tau_{o p}$ as well as the right tail of the bell shape curve shift to the left towards short time scales as $f_{m}$ increases. If we keep increasing $f_{m}$, the bell shape features in terms of the optimum time constant $\tau_{o p}$ and maximum amplitude disappear; the CWF decreases smoothly and rapidly from 1 to $1 / 2$ in time. This change in the behavior is governed by the condition of $\operatorname{arcosh}\left[\cos \left(\omega_{m} T\right)^{-1}\right]$ to stay a real positive quantity, which in turn requires $\cos \left(\omega_{m} T\right)$ being positive. This leads to a relationship between $T$ and $f_{m}$ for the CWF to keep its bell shape curve $f_{m} \leq 1 / 4 T=F / 4$. This behavior is reported in the inset of Fig $1(b)$.

We report in Fig 1(c) the variation of the optimum time constant $\tau_{o p}$ of the CWF as a function of $F$ and $f_{\mathrm{m}}$. As we mentioned above, $\tau_{o p}$ is almost insensitive to the variation of $F$ for a fixed $f_{m}$. We can see however that a slight dependence starts to appear when $f_{m}$ becomes a significant fraction of $F$; the mean value of $\tau_{o p}$ decreases as $f_{m}$ increases, and for high $f_{m}, \tau_{o p}$ increases slightly with $F$ to stagnate rapidly as $F$ becomes high. On the other hand, for a fixed $F, \tau_{o p}$ decreases enormously as $f_{m}$ increases. As we can see in Fig $1(\mathrm{c})$, there is a variation of almost four orders of magnitude in $\tau_{o p}$ when $f_{m}$ is varied over a $20 \mathrm{MHz}$ interval.
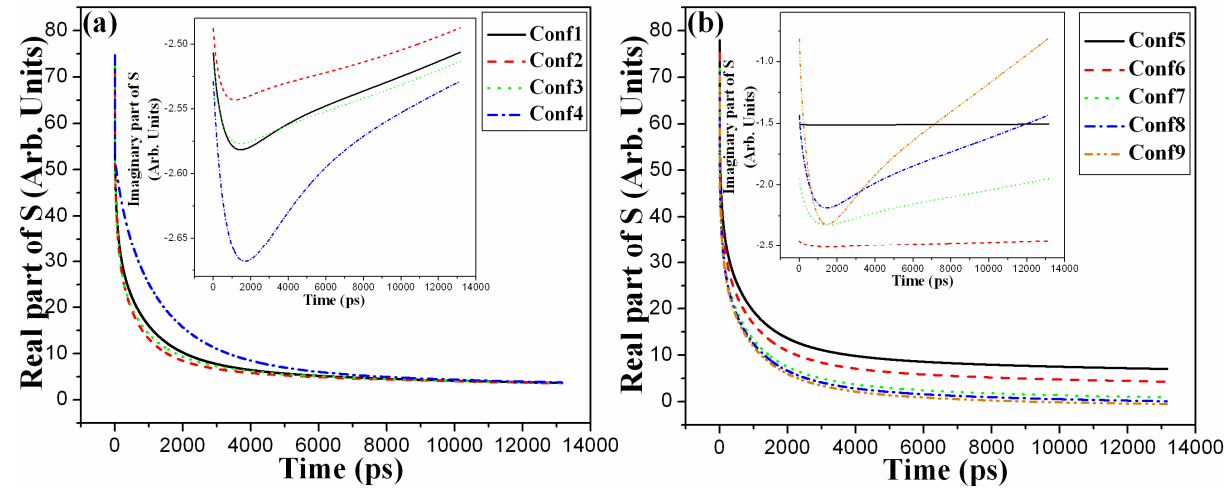

Figure 2 : Variation of the real parts and imaginary parts (insets) of the PPTTR signal as a function of time for sample configurations 1-4 (a) and 5-9 (b). 
To be able to validate the application of NID to the case of a laser pulse-train function excitation including the cumulative effect [10], we have considered mimicking a real situation of the PPTTR experiment using a time delay of $13157 p s$ with a $1 p s$ time resolution in measurement. The time delay corresponds to a laser repetition rate of $F=76 \mathrm{MHz}$ and is taken to be the time range of the transient thermal decay. We assume the sample to be an $80 \mathrm{~nm} \mathrm{Si} / \mathrm{SiGe}$ superlattice (SL) layer deposited on a semi-infinite Si substrate and covered by $30 \mathrm{~nm}$ thick $\mathrm{Al}$ film. The thickness of the sample is chosen small enough to satisfy the relation $t=1 / F=13157 p s \approx 12 \tau_{S L}$ [10], where $\tau_{S L}=d^{2} S L / \alpha_{S L}$ is the calculated time constant of the SL layer of thickness $d_{S L}$ and thermal diffusivity $\alpha_{S L}$. The thermal conductivity $\beta_{S L}$, thickness $d_{S L}, \mathrm{Al} / \mathrm{SL}$ interface thermal resistance $R_{K}$ and the external modulation frequency of the laser pump beam $f_{m}$ are varied to create 9 different configurations as shown in Table 1.

In Figs 2(a) and 2(b) we show the calculated real and imaginary parts of PPTTR signals for the different configurations as a function of time. The calculation is based on thermal Quadrupoles Method [13] and assumes a full 3D heat transport model where the Al film transducer is assumed to act as a thermal capacitor in which the temperature is spatially uniform.

Table 1 : Sample and PPTTR set up parameters used to simulate nine different configurations for the NID analysis.

\begin{tabular}{|c|c|c|c|c|c|}
\hline Configuration & $\begin{array}{c}\text { SL Layer } \\
\text { Thermal } \\
\text { conductivity } \\
(\mathrm{W} / \mathrm{mK})\end{array}$ & $\begin{array}{c}\text { SL layer } \\
\text { Thickness } \\
(\mathbf{n m})\end{array}$ & $\begin{array}{c}\mathrm{Al} / \mathrm{SL} \\
\text { Interface thermal } \\
\text { resistance } \\
\left(\mathrm{K} \cdot \mathrm{m}^{2} / \mathrm{W}\right)\end{array}$ & $\begin{array}{c}\text { Laser repetition } \\
\text { rate } \mathrm{F}(\mathrm{MHz})\end{array}$ & $\begin{array}{c}\text { Modulation } \\
\text { frequency } \\
f_{\mathrm{m}}(\mathrm{MHz})\end{array}$ \\
\hline 1 & 10 & 80 & \multirow{3}{*}{$10^{-9}$} & \multirow{9}{*}{76} & \multirow{4}{*}{1} \\
\hline 2 & 15 & 80 & & & \\
\hline 3 & 15 & 100 & & & \\
\hline 4 & \multirow{6}{*}{10} & \multirow{6}{*}{80} & $10^{-8}$ & & \\
\hline 5 & & & \multirow{5}{*}{$10^{-9}$} & & 0.1 \\
\hline 6 & & & & & 0.7 \\
\hline 7 & & & & & 5 \\
\hline 8 & & & & & 10 \\
\hline 9 & & & & & 20 \\
\hline
\end{tabular}
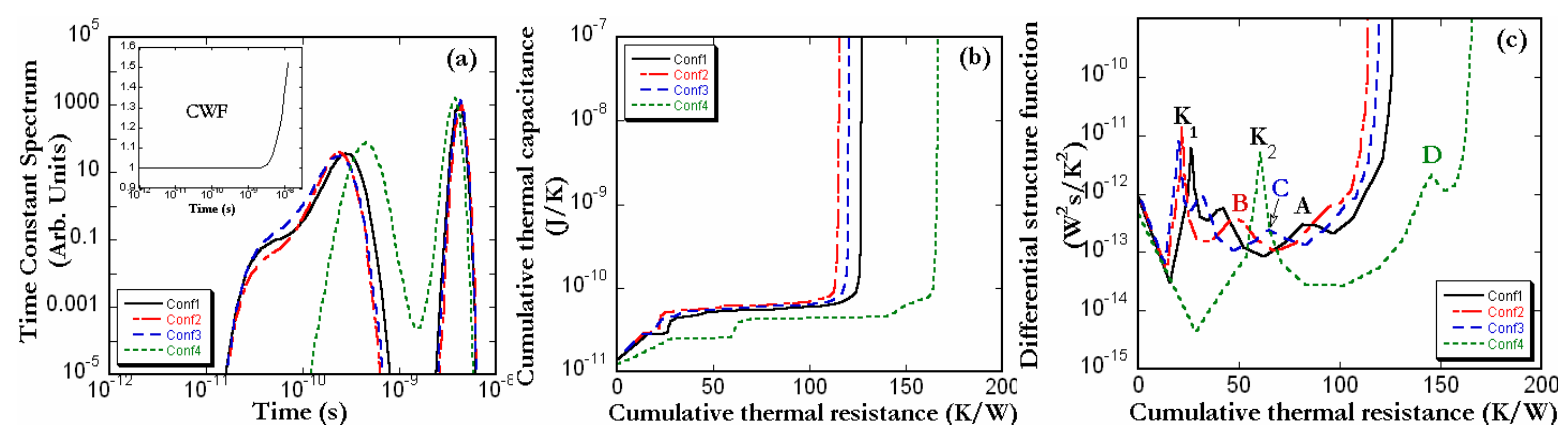

Figure 3 : Time Constant Spectrum function and CWF (inset) (a), Cumulative Structure Function (b) and Differential Structure Function (c) relative to the analysis by NID of the PPTTR thermal signals of configurations1-4. 
In order to use these graphs to identify the thermal properties of the layers in the structure, we apply modified NID method using mathematical transformations to include the cumulative effect [Eq. (2)]. The first step is the calculation of the Time Constant Spectrum (TCS).

The result is reported in Fig 3(a) for the first four configurations. As we can see in this figure, two main peaks are clearly distinguishable for each configuration; these peaks represent the dominant time constants in the response function and can be attributed to the SL layer including the $\mathrm{Al} / \mathrm{SL}$ interface thermal resistance $R_{K}$ and to the silicon substrate. The second step of the analysis consists in the evaluation of the cumulative structure function (CSF) and the differential structure function (DSF) referred as structure function in brief. We first plot the cumulative thermal capacitance $C_{\Sigma}$ as a function of the cumulative thermal resistance $R_{\Sigma}$ (CSF) along the heat flow path and then we plot the derivative of this graph (DSF). The results are reported in Figs 3(b) and 3(c), respectively. For each configuration, even though there is noise because of the deconvolution process in NID [10, 12], the DSF shows two distinguishable thermal resistance regions; two peaks that correspond to $R_{K}$ and to the SL layer. The peaks become even clearer as $\mathrm{R}_{\mathrm{K}}$ increases. Peaks $\mathrm{K}_{1}$ and $\mathrm{K}_{2}$ correspond to $R_{K}=10^{-9} \mathrm{~K} . \mathrm{m}^{2} / \mathrm{W}$ and $R_{K}=10^{-8} \mathrm{~K} . \mathrm{m}^{2} / \mathrm{W}$, respectively while peaks A, B, C and D refer to the SL layer. Because, PPTTR calculated signals assume arbitrary scaling factors and do not represent absolute temperature rises, a quantitative comparison between NID CSF and DSF data and the input data of thermal properties of the different layers, cannot be made, only a qualitative analysis can be done. The NID output results however, scale corresponding to the input data change. This shows that with a calibrated PPTTR signals, NID is able to distinguish the main thermal resistances and capacitances in the structure even when the cumulative effect due to the laser pulse train excitation is taken into account.

In the second series of configurations (5-9), we kept the same thermal and geometrical properties of the sample structure and we changed only the modulation frequency $f_{m}$ of the laser pump beam. We perform the same NID analysis to the calculated PPTTR signals. The results in terms of the CWF, TCS and DSF are reported in Figs 4(a), 4(b) and 4(c), respectively.
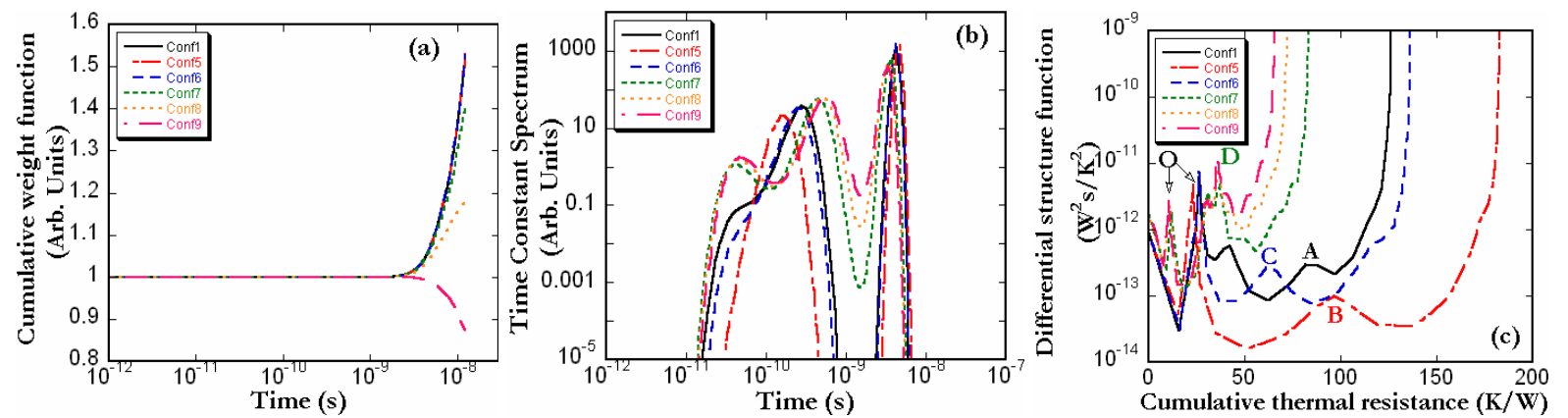

Figure 4 : Cumulative Weight Function CWF (a), Time Constant Spectrum Function TCS (b) and Differential Structure Function DSF (c) relative to the analysis by NID of the PPTTR thermal signals of configurations 1, 5-9.

Over the time range of the thermal transient $(t=13157 p s)$, Fig 4(a) shows that the CWF remains constant equals to 1 up to a delay of $\sim 1300 \mathrm{ps}$ and at higher delays either the CWF increases or decreases depending on $f_{m}$. After removing the effect of the CWF using the modified NID approach [Eq. (2)], the TCS reported in Fig 4(b) shows that as $f_{m}$ is increased, a third time constant starts to appear at the left of the main two time constants, and that all time constant seem to shift to shorter time scales. We see that for low $f_{m}$ (configuartions $1,5,6$ ), the far left 
time constant is almost negligible, and it tends to be more pronounced as $f_{m}$ gets higher to be a considerable fraction of the laser repetition rate $F$. Fig 4(c) reports the DSF curves corresponding to the TCS in Fig 4(b). We see here too that by increasing $f_{m}$, all characteristic peaks shift to the left and the total thermal resistance decreases. This last result is compatible with the reduction of the surface under the real part of the PPTTR signal by increasing $f_{m}$ as we can see in Fig 2(b). First, we tried to find a relation between $R_{t h}^{T}-R_{t h}^{S L}$ and $\Gamma / \beta_{S u b} \Sigma$, where $\Gamma=\sqrt{\alpha_{S u b} / \pi f_{m}}$ is the thermal penetration depth into the Si substrate whose thermal conductivity is $\beta_{S u b}$ and thermal diffusivity is $\alpha_{\text {Sub }}, R_{t h}^{T}$ the total thermal resistance, $R_{t h}^{S L}$ the thermal resistance corresponding to the SL layer (peaks A-D in Fig 4(c)), and $\Sigma$ is the laser pulse illuminated area. No clear correlation can be seen. Second, the shift to the left in the positions of the peaks in the TCS and consequently the thermal resistance peaks in the DSF, raise questions about the effect of the modulation frequency $f_{m}$ but at the same time shows how important $f_{m}$ is in a PPTTR experiment. The shift shows that depending on the sample, $f_{m}$ is to be chosen in certain interval for better extraction of the thermal properties. In fact, recent PPTTR measurement by Koh and Cahill [14] have shown a surprisingly frequency-dependence behavior of the thermal conductivity of semiconductor alloy materials when the modulation frequency $f_{m}$ is varied.

The variations in the features of the CWF as a function of $F$ and $f_{m}$ are a clear indicator on how the PPTTR's experimental parameters can affect the extraction of the thermal properties of the sample under study. From the CWF bell shape curve, we see that for a fixed $\left(F, f_{m}\right)$ pair, the intrinsic parameters of the PPTTR experiment influence the identification of the thermal resistances of the samples, specifically those whose time constants lie underneath the bell shape curve. While there is no effect at short time scales, the CWF introduces a factor of $1 / 2$ at long time scales. The most significant effect of the CWF occurs around $\tau_{o p}$ where the amplitude becomes much higher than 1 . This is the same factor that multiplies the peak amplitudes (main thermal resistances) of the TCS of the sample under study corresponding to the time constants around $\tau_{o p}$ [Eq. (3)]. A careful manipulation of the CWF such as the one proposed here using modified NID approach is needed in order to analysis more accurately the PPTTR data. The choice of $f_{m}$ plays a vital role in a PPTTR technique experiment. Of course the current study and the NID analysis have been developed in conventional diffusion (Fourier heat conduction) regime. If there are ballistic heat propagation effects, this further complicates the analysis of the PPTTR experiment. However, even in the latter case, the CWF could affect the identification of the "diffusive" component to the heat conduction equation and it deserves further analysis.

\section{CONCLUSIONS}

With the use of the mathematical tools of the Network Identification by Deconvolution (NID) method, a very interesting weight function that describes the intrinsic accumulation phenomenon in the Pump Probe Transient Thermoreflectance (PPTTR) technique in the homodyne configuration has been identified and introduced. This cumulative weight function (CWF) sheds light on how the PPTTR experimental parameters may affect the extraction of the thermal properties of the sample under study if they are not handled carefully. It brings to light once again the fundamental role of the modulation frequency $f_{m}$ for a correct operation of the PPTTR technique set up and an accurate analysis of the data. The CWF can be used to normalize the input data and NID could be applied. This is a powerful tool and an alternative way for the analysis of the thermal parameters in the PPTTR experiment. In the standard implementation, the 
exact temperature rise (i.e. calibration of the thermoreflectance coefficient and the input power) are needed. Future work will focus on the ballistic contributions to the heat transfer.

\section{REFERENCES}

1. C. A. Paddock, and G. L. Eesley, J. Appl. Phys, 60, 285 (1986).

2. D. G. Cahill, W. K. Ford, K. E. Goodson, G. D. Mahan, A. Majumdar, H. J. Maris, R. Merlin, and S. R. Phillpot. J. Appl. Phys, 93, 793 (2003).

3. A. Schmidt, M. Chiesa, X. Chen and G. Chen, Rev. Sci. Instrum, 79, 064902 (2008).

4. D. G. Cahill, Rev. Sci. Instrum, 61, 802 (1990).

5. Y. Ezzahri, S. Grauby, S. Dilhaire, J. M. Rampnoux, and W. Claeys, J. Appl. Phys, 101, 013705 (2007).

6. S. Dilhaire, W. Claeys, J. M; Rampnoux, and C. Rossignol, “Optical Heterodyne Sampling Device", Patent, WO/2007/045773 (2007).

7. W. S. Capinski and H. J. Maris, Rev. Sci. Instrum, 67, 2720 (1996).

8. D. G. Cahill, Rev. Sci. Instrum, 75, 5119 (2004).

9. A. J. Schmidt, X. Chen and G. Chen, Rev. Sci. Instrum, 79, 114902 (2008).

10. Y. Ezzahri and A. Shakouri, Rev. Sci. Instrum, 80, 074903 (2009).

11. R. J. Stevens, A. N. Smith and P. M. Norris, Rev. Sci. Instrum, 77, 084901 (2006).

12. V. Székely and T. V. Bien, Solid-State Electronics, 31, 1363 (1988).

13. D. Maillet, S. André, J. C. Batsale, A. Degiovanni, and C. Moyne, Thermal Quadrupoles: Solving the Heat Equation Through Integral Transforms Wiley, Chichester, West Sussex, UK, 2000.

14. Y. K. Koh and D. G. Cahill, Phys. Rev. B, 76, 075207 (2007). 